\title{
Application of Discrete Wavelet Transform for Differential Protection of Power Transformers
}

\author{
Mario Orlando Oliveira' ${ }^{1,2}$ and Arturo Suman Bretas ${ }^{1}$ \\ ${ }^{1}$ Electrical Engineering Department, Federal University of Rio Grande do Sul \\ (UFRGS) \\ ${ }^{2}$ Energy Studies Center (CEED), National University of Misiones \\ (UNaM) \\ ${ }^{1}$ Brazil \\ ${ }^{2}$ Argentina
}

\section{Introduction}

Power transformers (PT) play an extremely important role on the reliability and energy supply continuity of Electric Power Systems (EPS). The inherent characteristic of power transformers introduce a number of unique problems that are not present in the protection of transmission lines, generators, motors or other power system apparatus (Horowitz \& Phadke, 2008). When PT internal faults occur, immediate disconnection of the faulted transformer is necessary to avoid extensive damage and/or preserve power system stability and power quality (Harlow, 1999). Currently, percentage differential protection is a common practice for power transformer protection. However, nonlinearities in the transformer core, the current transformer (CT) core or in both, cause a substantial differential current to flow when there is no fault. Thus, these false differentials currents can cause a percentage differential relay miss trip. To mitigate some of these problems the differential relays are equipped with harmonic restraint, where the magnitudes of the second and fifth harmonic component are compared with the fundamental frequency component to discriminate internal faults from magnetizing inrush currents and transformer over-excitation, respectively (Anderson, 1999). However, performance limitations are still reported even for such phenomena. In order to overcome such limitation, a significant number of relaying formulations have been proposed (Abed \& Mohammed, 2007; Eissa, 2005; Faiz \& Lotfi-Fard, 2006; Mao \& Aggarwal, 2000; Megahed et al., 2008; Morate \& Nicoletti, 1999; Ngaopitakkul \& Kunakorn, 2006; Saleh \& Rahman, 2005. Thomas \& Ozgönenel, 2007; Wang \& Butler, 2001; Wiszniewski \& Kasztenny, 1995; Zaman et al., 1996). These formulations are based on finite elements, artificial neural networks, fuzzy systems, dynamical principal components analysis, wavelet transforms (WTs) and hybrid systems. However, all mentioned relaying formulations have hard to design parameters, which make real life construction difficult.

In detecting faults in EPS and, specifically PT, frequency analysis is required so that the transient signal components can be isolated. This process helps to identify particular 
phenomena that generated the transient signals. It should be noted that the waveforms associated with electromagnetic transients are typically non-periodic in nature, containing both high-frequency oscillations as short duration pulses superimposed on low frequency signals. Still, need to know the fault occurrence instant encourages the application of techniques with precise time and frequency resolution.

In this chapter, a novel percentage differential relaying algorithm for three-phase power transformers protection based on Discrete Wavelet Transforms (DWT) is presented. The proposed algorithm's formulation uses logical decision criteria based on wavelets coefficient spectral energy variation to identify and discriminate correctly external faults, inrush currents and incipient internal transformer faults. In order to analyze the proposed algorithms efficiency, it was built in MATLAB ${ }^{\circledR}$ platform (Matlab, 2010) and tested with simulated fault cases under BPA's ATP/EMTP software (ATP/EMTP, 2002).

\section{Wavelet Transform (WT)}

The Wavelet Transform (WT) theory is based on signal analysis using varying scales in the time and frequency domain. Formalization was carried out in the 80s, based on the generalization of familiar concepts. The wavelet term was introduced by French geophysicist Jean Morlet. The seismic data analyzed by Morlet exhibit frequency component that changed rapidly over time, for which the Fourier Transform was not appropriate as an analysis tool. Thus, with the help of theoretical physicist Croatian Alex Grossmann, Morlet introduced a new transform which allows the high-frequency events identification with a better temporal resolution (Polikar, 1999).

Faulted EPS signals are associated with fast electromagnetic transients, are typically nonperiodic and with high-frequency oscillations. This characteristic present a problem for traditional Fourier analysis because its assumes a periodic signal and a wide-band signal requires more dense sampling and longer time periods to maintain good resolution in the low frequencies (Robertson et al., 1996). Thus WT is a powerful tool in the power system transient phenomena analysis. It has the ability to extract information from the transient signals simultaneously in both time and frequency domains and has replaced the Fourier analysis in many applications (Phadke \& Thorp, 2009).

\subsection{Continuous Wavelet Transform (CWT)}

The Short-Time Fourier Transform (STFT) of the continuous signal $x(t)$, can be seen as the Fourier Transform (FT) of the signal with windowed $x(t) \cdot g(t-\tau)$ or also as a signal decomposition $x(t)$ into basis functions $g(t-\tau) \cdot e^{-j w t}$. The function based term refers to a complete set of functions that, when combined the sum with specific weight can be used to construct the signal (Bentley \& McDonnell, 1994).

In the FT case the base function are complex sinusoid $e^{-j w t}$ with a windows centred on $\tau$ time. The WT is described in terms of its basic functions, called wavelet or mother wavelet, and variable frequency $w$ is replaced by an ever-escalating variable factor $a$ (which represents the swelling) and generally to variable displacement in time $\tau$ is represented by $b$.

The main characteristic of the WT is that it uses a variable window to scan the frequency spectrum, increasing the temporal resolution of the analysis. The wavelets are represented by: 


$$
\psi_{a, b}(t)=\frac{1}{\sqrt{a}} \cdot \psi\left(\frac{t-b}{a}\right)
$$

In the equation (1), the constant $1 / \sqrt{a}$ is used to normalize the energy and ensure that the energy of $\psi_{a, b}(t)$ is independent of the dilation level (Simpson, 1993). The wavelet is derived from operations such as dilating and translating the mother wavelet, $\psi$, which must satisfy the admissibility criterion given by (Daubechies, 1990):

$$
C_{\psi}=\int_{-\infty}^{+\infty} \frac{|\widehat{\psi}(y)|^{2}}{|y|} d y \leq \infty
$$

where $\hat{\psi}(y)$ is the FT of $\psi(t)$. This means that if $\widehat{\psi}$ is a continuous function, then $C_{\psi}$ is finite only if $\psi(0)=0$, ie (Daubechies, 1990):

$$
\int_{-\infty}^{+\infty} \psi(t) d t=0
$$

Thus, it is evident that WT has a zero rating, property that increases the degrees of freedom, allowing the introduction of the dilation parameter of the window (Sarkar \& Su, 1998).

The Continuous Wavelet Transform (CWT) of the continuous signal $x(t)$ is defined as:

$$
(C W T)(a, b)=\int_{-\infty}^{+\infty} x(t) \cdot \psi_{a . b}(t) d t=\frac{1}{\sqrt{a}} \int_{-\infty}^{+\infty} x(t) \cdot \psi\left(\frac{t-b}{a}\right) d t
$$

where the scale factor $a$, and the translation factor $b$ are continuous variables.

The WT coefficient is an expansion and a particular shift represents how well the original signal $x(t)$ corresponds to the translated and dilated mother wavelet. Thus, the coefficient group of $\operatorname{CWT}(a, b)$ associated with a particular signal is the wavelet representation of the original signal $x(t)$ in relation to the mother wavelet (Aggarwal \& Kim, 2000).

\subsection{Discrete Wavelet Transform (DWT) 2.2.1 Why is DWT needed?}

Although the discretized continuous wavelet transform enables the computation of the continuous wavelet transform by computers, it is not a true discrete transform. As a matter of fact, the wavelet series is simply a sampled version of the CWT, and the information it provides is highly redundant as far as the reconstruction of the signal is concerned. This redundancy, on the other hand, requires a significant amount of computation time and resources. The Discrete Wavelet Transform (DWT), on the other hand, provides sufficient information both for analysis and synthesis of the original signal, with a significant reduction in the computation time. The DWT is considerably easier to implement when compared to the CWT. The basic concepts of the DWT will be introduced in this section along with its properties and the algorithms used to compute it (Polikar, 1999).

\subsubsection{DWT definition}

The redundancy of information and the enormous computational effort to calculate all possible translations and scales of CWT restricts its use. An alternative to this analysis is the 
discretization of the scale and translation factors, which leads to the DWT. There are several ways to introduce the concept of DWT, the main are the decomposition bands and the decomposition pyramid (or Multi-Resolution Analysis -MRA), developed in the late 70's (Rioul \& Vetterli, 1991). The DWT of the continuous signal $x(t)$ is given by:

$$
(D W T)(m, p)=\int_{-\infty}^{+\infty} x(t) \cdot \psi_{m, p} d t
$$

where $\psi_{m, p}$ form wavelet function bases, created from a translated and dilated mother wavelet using the dilation $m$ and translation $p$ parameters, respectively.

Thus, $\psi_{m, p}$ is defined as:

$$
\psi_{m, p}=\frac{1}{\sqrt{a_{0}^{m}}} \psi\left(\frac{t-p b_{0} a_{0}^{m}}{a_{0}^{m}}\right)
$$

The DWT of a discrete signal $x[n]$ is derived from CWT and defined as (Aggarwal \& Kim, 2000):

$$
(D W T)(m, k)=\frac{1}{\sqrt{a}} \sum_{n} x[n] \cdot g\left(\frac{k-n b_{0} a_{0}^{m}}{a_{0}^{m}}\right)
$$

where $g\left(^{*}\right)$ is the mother wavelet and $x[n]$ is the discretized signal.

The mother wavelet may be dilated and translated discretely by selecting the scaling and translation parameters $a=a_{0} \mathrm{~m}$ and $b=n b_{0} a_{0}{ }^{m}$ respectively (with fixed constants $\mathrm{a}_{0}>1, \mathrm{~b}_{0}>1, m$ and $n$ belonging the set of positive integers).

\subsection{Multi-Resolution Analysis (MRA)}

The problems of temporal and frequency resolution found in the analysis of signals with the STFT (best resolution in time at the expense of a lower resolution in frequency and viceversa) can be reduced through a Multi-Resolution Analysis (MRA) provided by WT. The temporal resolutions, $\Delta t$, and frequency, $\Delta f$, indicate the precision time and frequency in the analysis of the signal. Both parameters vary in terms of time and frequency, respectively, in signal analysis using WT. Unlike the STFT, where a higher temporal resolution could be achieved at the expense of frequency resolution. Intuitively, when the analysis is done from the point of view of filters series, the temporal resolution should increase increasing the center frequency of the filters bank. Thus, $\Delta f$ is proportional to $f$, ie:

$$
\frac{\Delta f}{f}=c
$$

where $c$ is constant.

The main difference between DWT and STFT is the time-scaling parameter. The result is geometric scaling, i.e. $1,1 / a, 1 / a^{2}, \ldots$; and translation by $0, n, 2 n$, and so on. This scaling gives the DWT logarithmic frequency coverage in contrast to the uniform frequency coverage of the STFT, as compared in Fig. 1.

The CWT follows exactly these concepts and adds the simplification of the scale, where all the impulse responses of the filter bank are defined as dilated versions of a mother wavelet 
(Rioul \& Vetterli, 1991). The CWT is a correlation between a wavelet at different scales and the signal with the scale (or the frequency) being used as a measure of similarity. The CWT is computed by changing the scale of the analysis window, shifting the window in time, multiplying by the signal, and integrating over all times. In the discrete case, filters of different cut-off frequencies are used to analyze the signal at different scales. The signal is passed through a series of high pass filters to analyze the high frequencies, and it is passed through a series of low pass filters to analyze the low frequencies. Thus, the DWT can be implemented by multistage filter bank named MRA (Mallat, 1999), as illustrated on Fig. 2. The Mallat algorithm consists of series of high-pass and the low-pass filters that decompose the original signal $x[n]$, into approximation $\mathrm{a}(\mathrm{n})$ and detail $\mathrm{d}(\mathrm{n})$ coefficient, each one corresponding to a frequency bandwidth.

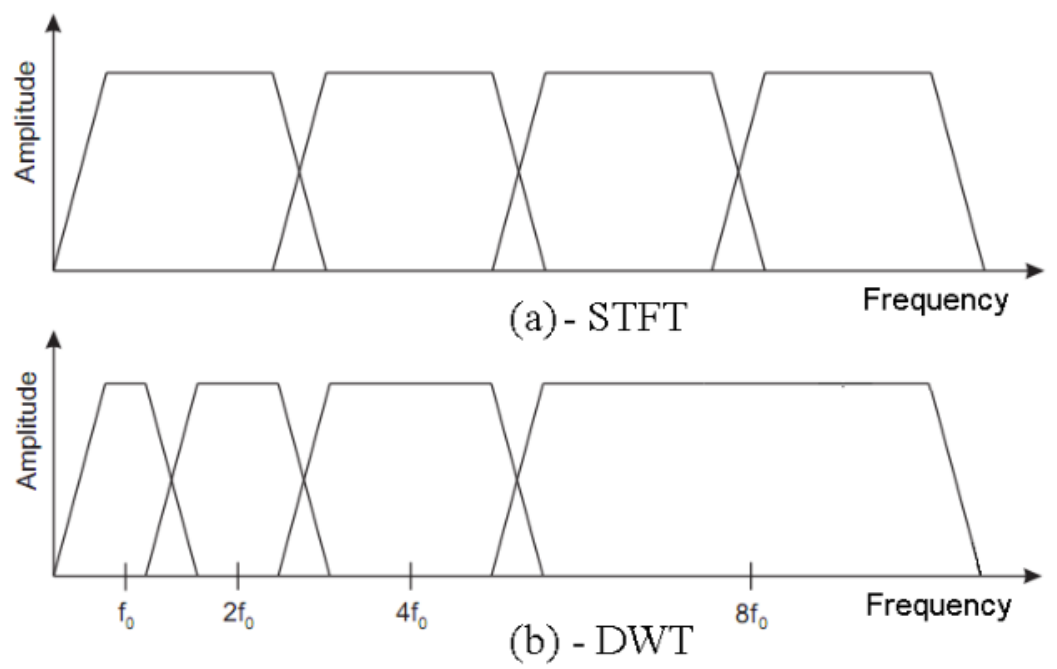

Fig. 1. Comparison of (a) the STFT uniform frequency coverage to (b) the logarithmic coverage of the DWT.

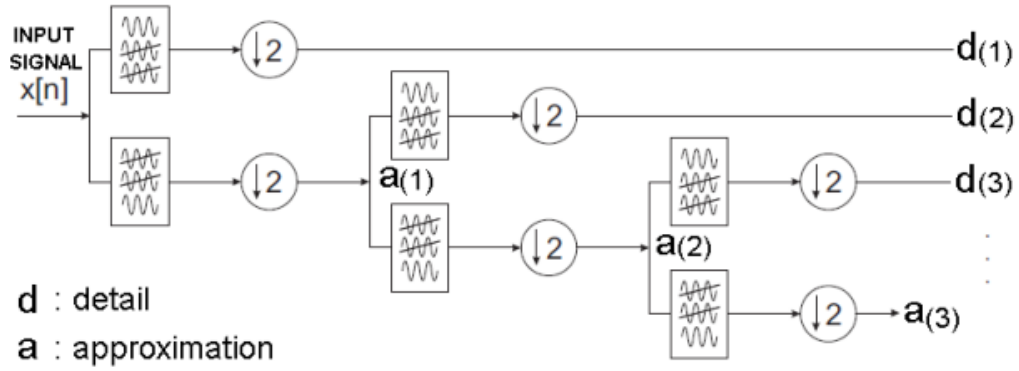

Low-Pass Filter High-Pass Filter Downsampling by 2

Fig. 2. DWT filter bank framework. 
The resolution of the signal, which is a measure of the amount of detail information in the signal, is changed by the filtering operations, and the scale is changed by up-sampling and down-sampling (sub-sampling) operations. Sub-sampling a signal corresponds to reducing the sampling rate, or removing some of the samples of the signal. On the other hand, upsampling a signal corresponds to increasing the sampling rate of a signal by adding new samples to the signal.

The procedure starts with passing this signal $x[n]$ through a half band digital low-pass filter with impulse response $h[n]$. The filtering process corresponds to the mathematical operation of signal convolution with the impulse response of the filter. The convolution operation in discrete time is defined as follows (Polikar, 1999):

$$
x[n] * h[n]=\sum_{k=-\infty}^{\infty} x[k] \cdot h[n-k]
$$

A half band low-pass filter removes all frequencies that are above half of the highest frequency in the signal. For example, if a signal has a maximum of $1000 \mathrm{~Hz}$ component, then half band low-pass filtering removes all the frequencies above $500 \mathrm{~Hz}$. However, it should always be remembered that the frequency unit for discrete time signals is radians.

After passing the signal through a half band low-pass filter, half of the samples can be eliminated according to the Nyquist's rule. Simply discarding every other sample will subsample the signal by two, and the signal will then have half the number of points. The scale of the signal is now doubled. Note that the low-pass filtering removes the high frequency information, but leaves the scale unchanged. Only the sub-sampling process changes the scale. Resolution, on the other hand, is related to the amount of information in the signal, and therefore, it is affected by the filtering operations. Half band low-pass filtering removes half of the frequencies, which can be interpreted as losing half of the information. Therefore, the resolution is halved after the filtering operation. Note, however, the sub-sampling operation after filtering does not affect the resolution, since removing half of the spectral components from the signal makes half the number of samples redundant anyway. Half the samples can be discarded without any loss of information.

This procedure can mathematically be expressed as (Polikar, 1999):

$$
y[n]=\sum_{k=-\infty}^{\infty} h[k] \cdot x[n-k]
$$

The decomposition of the signal into different frequency bands is simply obtained by successive highpass and lowpass filtering of the time domain signal. The original signal $x[n]$ is first passed through a halfband highpass filter $g[n]$ and a lowpass filter $h[n]$. After the filtering, half of the samples can be eliminated according to the Nyquist's rule, since the signal now has a highest frequency of $p / 2$ radians instead of $p$. The signal can therefore be sub-sampled by 2 , simply by discarding every other sample. This constitutes one level of decomposition and can mathematically be expressed as follows (Polikar, 1999):

$$
y_{\text {high }}[k]=\sum_{n} x[n] \cdot g[2 k-n]
$$




$$
y_{\text {low }}[k]=\sum_{n} x[n] \cdot h[2 k-n]
$$

where $y_{h i g h}[k]$ and $y_{\text {low }}[k]$ are the outputs of the high-pass and low-pass filters, respectively, after sub-sampling by 2 .

\subsection{Energy and power of discrete signal}

The total energy of a discrete signal $x[n]$ is given for equation (Haykin \& Veen, 2001):

$$
E=\sum_{n=-\infty}^{+\infty} x^{2}[n]
$$

and the average power is defined as:

$$
P=\lim _{x \rightarrow \infty} \frac{1}{2 N} \sum_{n=-N}^{N} x^{2}[n]
$$

For a periodic signal of fundamental period $N$, the average power is given by:

$$
P=\frac{1}{N} \sum_{n=0}^{N-1} x^{2}[n]
$$

\section{Differential protection of power transformers using DWT}

\subsection{Percentage differential protection}

Differential protection schemes are widely used by electric companies to protect EPS equipments. This relaying technique is applied on power transformers protection, buses protection, and large motors and generators protection among others (Anderson, 1999). Considering power transformers rated above $10 \mathrm{MVA}$, the percentage differential relay with harmonic restraint is the most used protection scheme (Horowitz \& Phadke, 2008). The percentage differential relay can be implemented with an over-current relay (R) and operation (o) and restriction coils (r), as illustrated on Fig. 3, connected between Current Transformer (CTs).

Under normal operating conditions or external faults, the CTs secondary currents, $i_{2 P}$ and $i_{2 S}$, have close absolute values. The differential protection formulation compares the differential current to a fixed threshold value. To include CTs transformation errors, CTs mismatch and power transformer variable taps, the differential current $\left(i_{d}\right)$ can be compared to a fixed percentage value of the restraint current $\left(i_{r}\right)$. This percentage characteristic of the relay, named $K$, is given by:

$$
K=\frac{i_{2 P}-i_{2 S}}{\left(i_{2 P}+i_{2 S}\right) / 2}=\frac{i_{d}}{i_{r}}
$$

The relay identifies an internal fault when the differential current exceeds the percentage value $K$ of the restraint current, where $i_{o p}$ is the operation current of relay:

$$
i_{d}=i_{o p} \geq K \cdot i_{r}=K \cdot \frac{\left(i_{2 P}+i_{2 S}\right)}{2}
$$




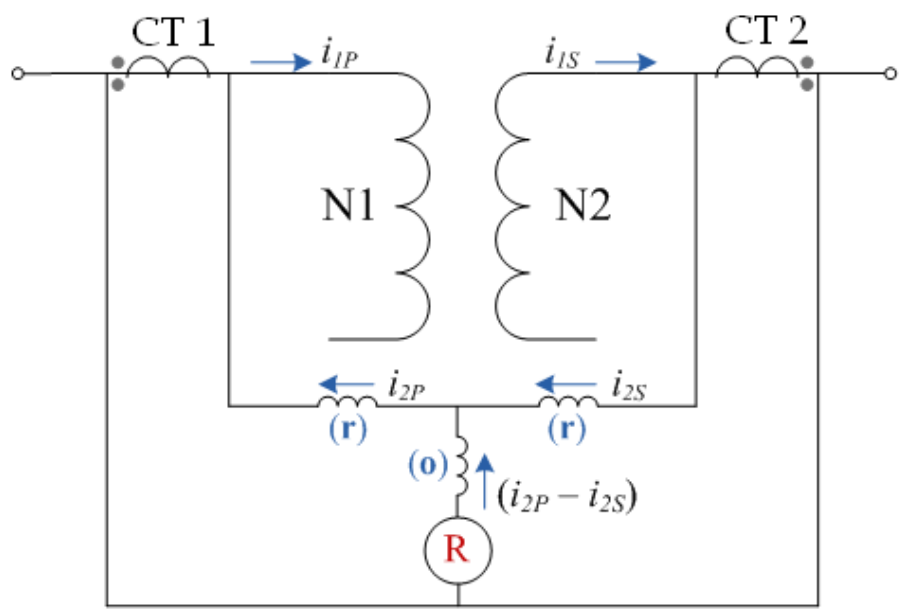

Fig. 3. Differential relay connections.

\subsection{Proposed protection algorithm using DWT}

A change in the spectral energy of the wavelets components of the current differential is noted when different electrical events (external faults, internal faults and/or inrush current) occur on the power transformers (Megahed et al., 2008). In this sense, the discrimination criterion of the proposed protection algorithm in this work is based in the spectral energy level generated by the electrical event type. The flow chart of the proposed algorithm is presented on Fig. 4.

In the disturbance detection (BLOCK 1) the activation current is calculated. The activation current is calculated for each phase through the percentage characteristic $K$ and the restraint currents showed in equation (17). The activation current is given by:

$$
\left|I d_{A, B, C}\right| \geq\left|I_{a}\right|=\left|k \cdot i_{r}\right|=\left|K \cdot \frac{\left(i_{2 p}+i_{2 s}\right)^{A, B, C}}{2}\right|
$$

where $I_{a}$ is the activation current, $I d_{A, B, C}$ is the differential current on A, B and C phases, $K$ is the percentage differential characteristic and $i_{r}$ is the restraint current.

In the disturbance identification (BLOCK 2) the three-phase differential currents are initially processed through a DWT implemented as filter bank. After, a restraint index $R_{\text {ind }}$, is calculated. This index quantifies the relative magnitude characteristic of the differential signals in the $1^{\text {st }}$ detail (D1) and is defined as the relation between the maximum detail coefficient from D1 and the detail-spectrum-energy (DSE) of the wavelet coefficient. Thus, $R_{\text {ind }}$ is given by:

$$
R_{\text {ind }}=\frac{d_{\max , D 1}}{\sum_{c=1}^{M}\left|d_{(c)}\right|^{2} \Delta t}
$$


where $d_{\max , D 1}$ is the maximum detail coefficient from $D 1, M$ is the total number of wavelet coefficients from $D 1$ and $\Delta t$ is the sampling period.

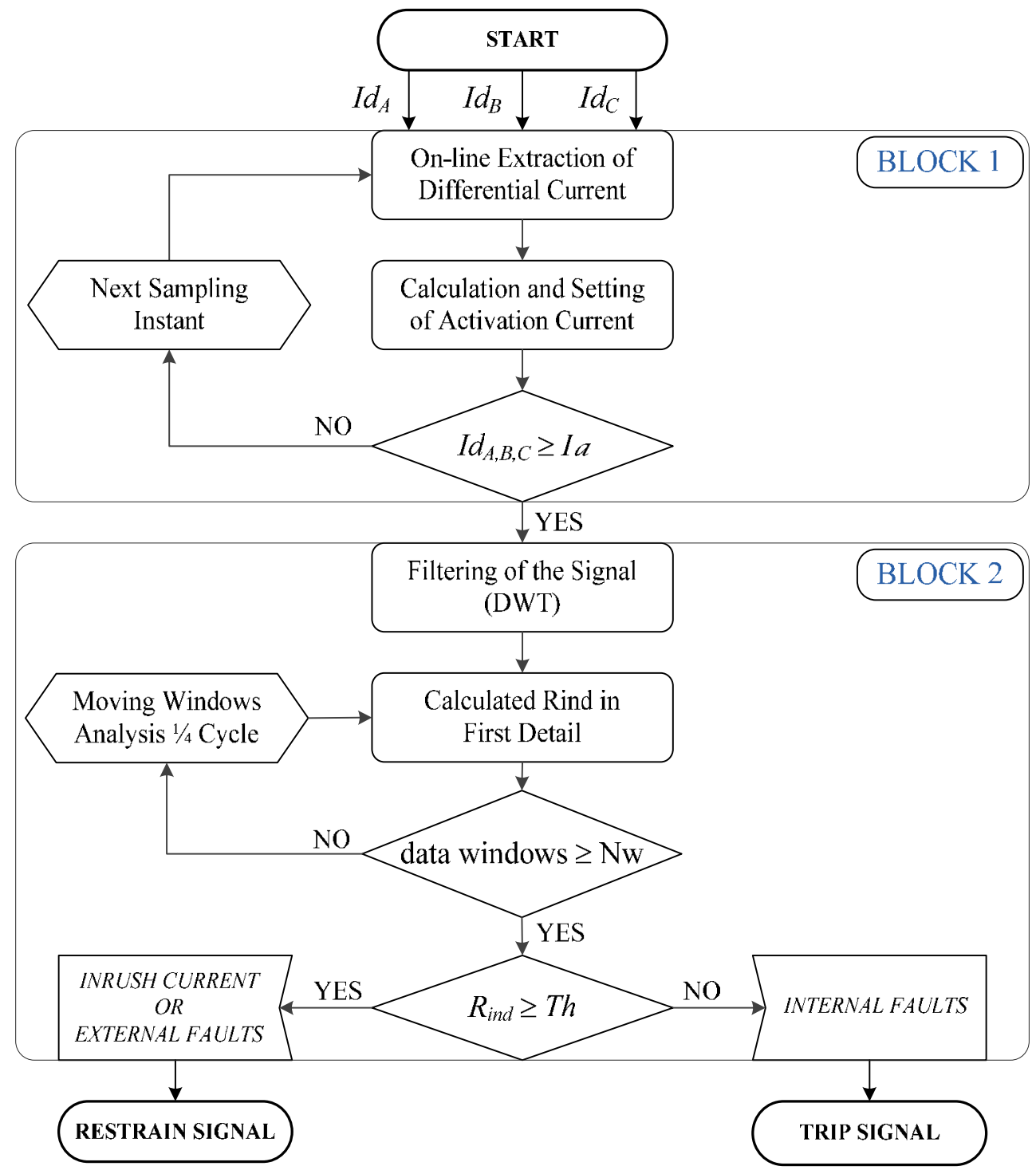

Fig. 4. Proposed Algorithm's Operation Scheme.

The proposed algorithm was implemented in MATLAB® platform (Matlab, 2010). Fig. 5 presents the graphical interface developed with three input block: 1) selecting the disturbance type; 2) selecting of the wavelet analysis characteristics; 3) analyzed results outputs. 


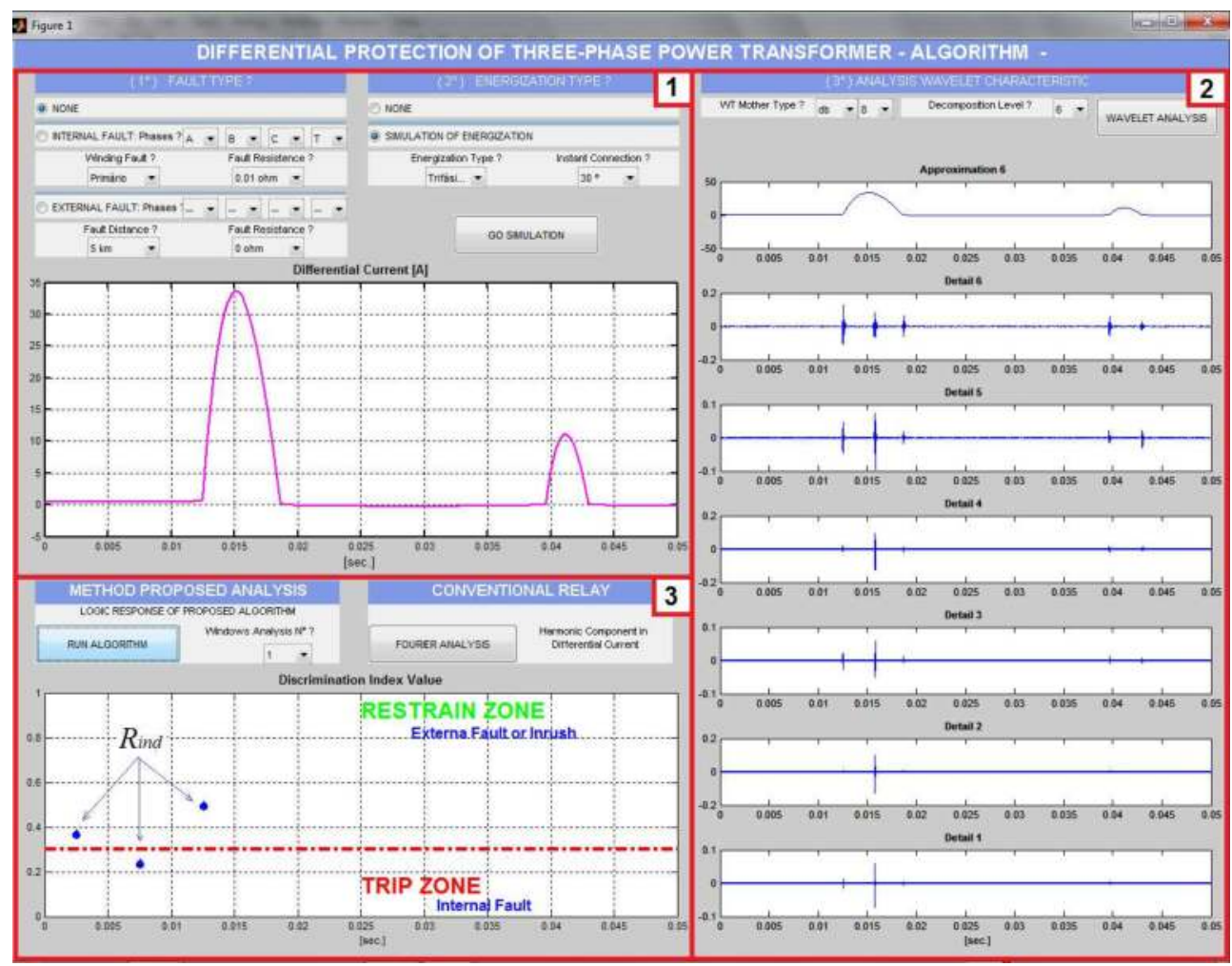

Fig. 5. Graphical implementation in MATLAB® environment.

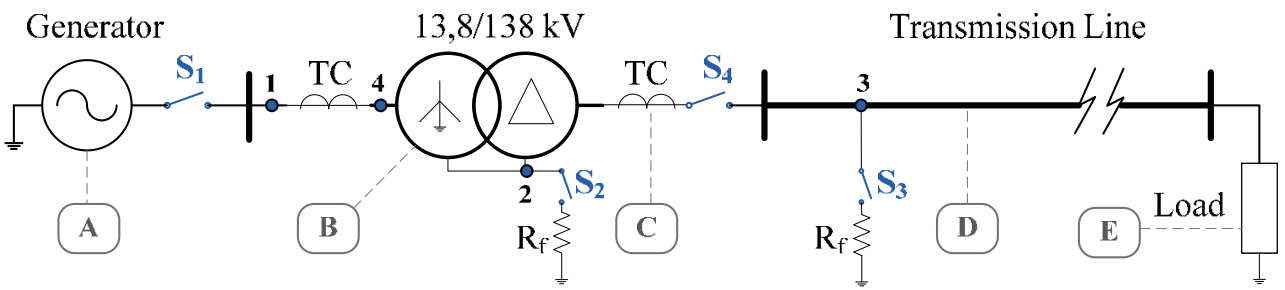

Fig. 6. Simulated electric power system.

\section{Case study}

Fig. 6. illustrates the studied electrical power system. The studied system consists of:

a. Generator: $13.8 \mathrm{kV}, 30 \mathrm{MVA}, 50 \mathrm{~Hz}$;

b. $\quad$ Power Transformer (PT): $35 \mathrm{MVA}, 13.8 / 138 \mathrm{kV}, \mathrm{Yg}-\Delta$;

c. Current Transformers (CT) with $1200 / 5$ and $200 / 5$ turns ratio; 
d. Transmission line: with a length of $100 \mathrm{~km}$;

e. Variable Load of 3, 10 or 25 MVA all with a 0.92 power factor.

The switches shown in Fig. $6, S_{1}$ and $S_{4}$, are used to simulate the energization operation of the PT. In this phenomenon the transformer is connected without load. The switch $S_{3}$ simulates external faults through a fault resistor $R_{f}$. The closing of the switch $S_{2}$ simulates an internal faults to the PT in both the primary and secondary windings.

\subsection{Types of analyzed events}

The proposed algorithm operates through three-phase differential currents. The simulations performed are presented on Table 1:

\begin{tabular}{|c|l|}
\hline \hline$N^{\circ}$ & \multicolumn{1}{|c|}{ Type Event } \\
\hline \hline $\mathbf{1}$ & $\begin{array}{l}\text { Different energization cases, comprising different switching inception angles }\left(0^{\circ},\right. \\
\left.30^{\circ}, 60^{\circ} \text { and } 90^{\circ}\right) \text { by closing the switch } \mathrm{S}_{1} \text { in the Low Voltage }(\mathrm{LV}) \text { side. }\end{array}$ \\
\hline $\mathbf{2}$ & $\begin{array}{l}\text { Internal faults in both primary and secondary sides of the transformer. These faults } \\
\text { were simulated with a fault resistance } \mathrm{R}_{\mathrm{f}} \text { values of } 0 \Omega, 0.01 \Omega, 10 \Omega \text {, and } 100 \Omega .\end{array}$ \\
\hline $\mathbf{3}$ & $\begin{array}{l}\text { Several cases of external faults with fault resistances } \mathrm{R}_{\mathrm{f}} \text { values: } 0 \Omega, 0.01 \Omega, 10 \Omega, \text { and } \\
100 \Omega .\end{array}$ \\
\hline $\mathbf{4}$ & Faults applied between the PT and the CTs. \\
\hline $\mathbf{5}$ & Energizing the PT with the presence of internal faults \\
\hline $\mathbf{6}$ & Energizing the PT with the presence of external faults. \\
\hline
\end{tabular}

Table 1. Simulated Events.

\section{Simulation and analysis result}

In order to evaluate the proposed protection algorithm efficiency, internal faults and transient inrush currents have been simulated. For each simulation, the proposed algorithm used different mother wavelets to evaluate accuracy and speed. The mother wavelets tested in this study were: Daubechies (Db), Symlet (Sy), Haar (Hr), Coiflet (Coif) and Morlet (Mo).

\subsection{Transient signal and fault current simulation}

The transient signal (inrush current) and fault current simulated are concentrated in the following situations:

- $\quad$ Fig. 7 presents an energization case. Part (a) illustrates the voltages in the secondary side of the PT. Part (b) the differential current are presented.

- Fig. 8 illustrates a case of energization with internal fault (concurrent event). The internal fault was simulated in the A phase with fault resistance $R_{f}=10 \Omega$.

- Fig. 9 illustrates a case of external fault removal. The faults occurring at $3 \mathrm{~km}$ to the PT on the transmission line. 


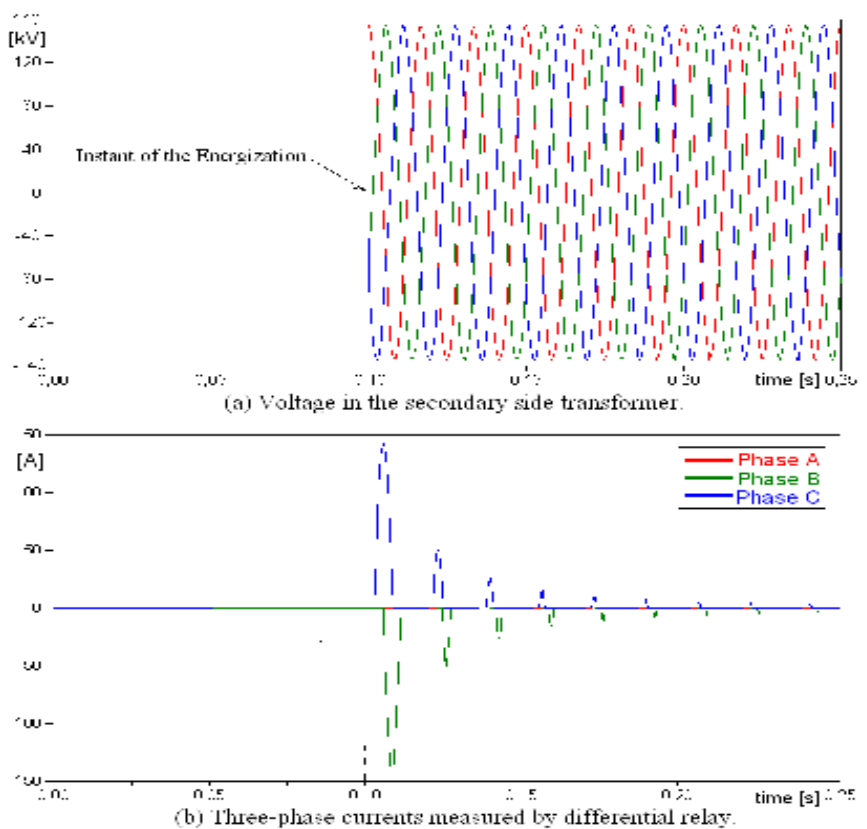

Fig. 7. Energization simulation on PT.

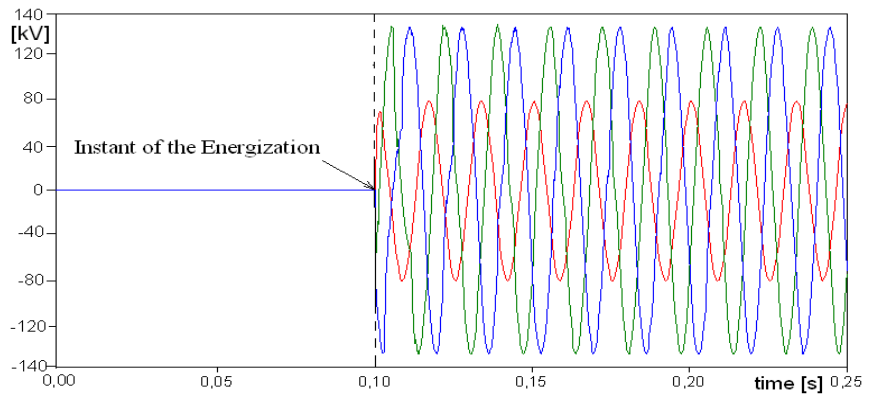

(a) Voltage in the secondary side transformer.

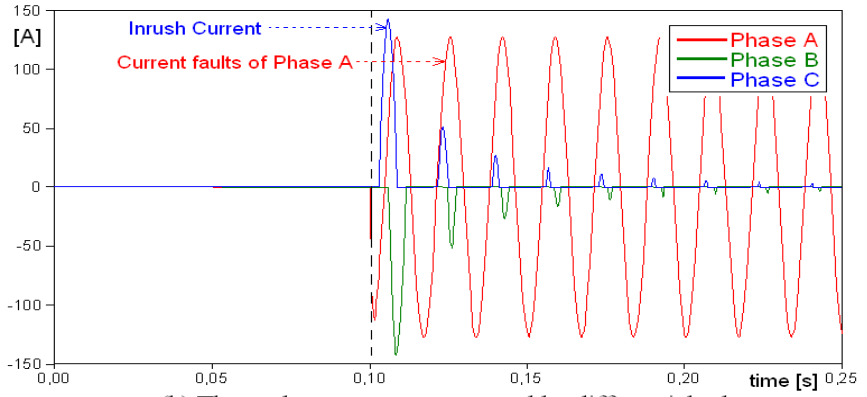

(b) Three-phase currents measured by differential relay.

Fig. 8. Energization and internal faults simulation on PT. 


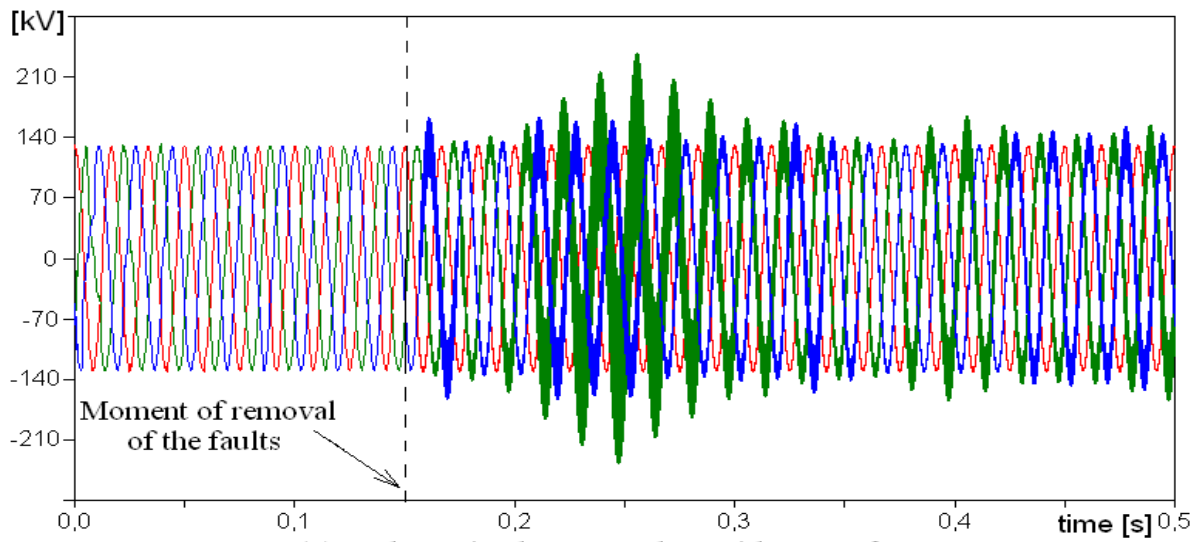

(a) Voltage in the secondary side transformer.

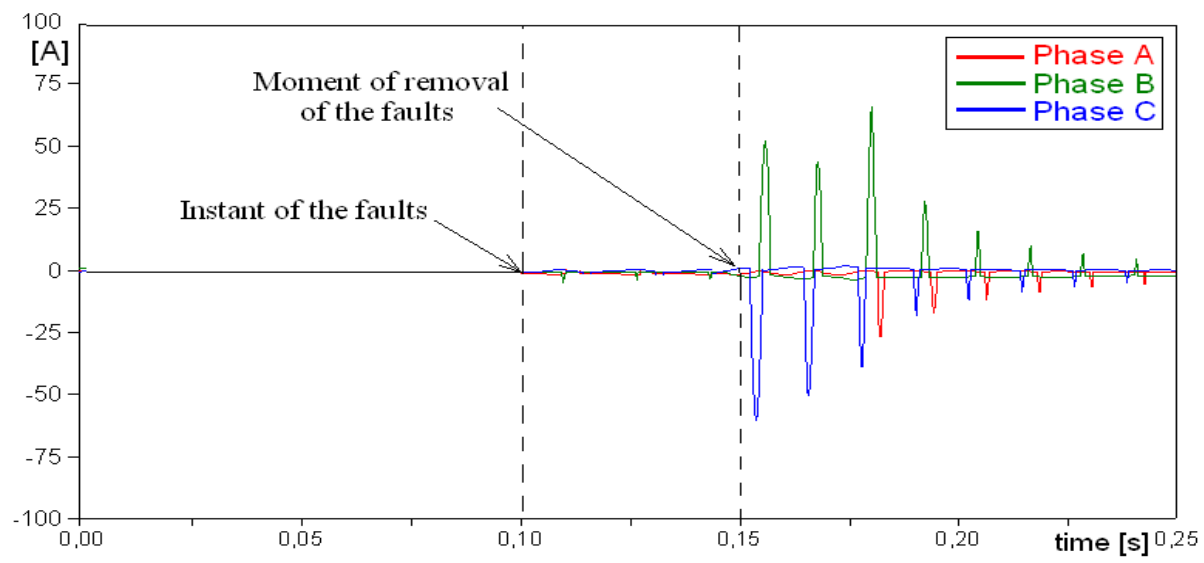

(b) Three-phase currentes measured by differential relay.

Fig. 9. External faults removal simulation.

\subsection{Algorithm proposed analysis}

Depending on the voltage angle in which the transformer is connected to the EPS, its residual flux can cause transient inrush currents which are correctly discriminated by the proposed protection algorithm.

Fig. 10 shows the algorithm response to a transient inrush current. Fig. 10(a) presents the inrush current in differential circuit of the power transformer. Fig. 10(b) shows the first detail of the DWT decomposition where a maximum number of three windows analyses are implemented on detail coefficient of the WT. Three windows analyses $\left(\mathrm{N}_{\mathrm{W}}\right)$ are necessary to guarantee a correct decision by the methodology. The window analysis is moving $1 / 4$ cycle for each restraint index $\left(R_{\text {ind }}\right)$ calculated to avoid false operations of the protection algorithm. After calculating and analyzing the ratio index for event discrimination, the proposed algorithm sends a restrain signal to the protection relay. Note on Fig. 10(c) the adaptive threshold value is proportional to the differential current caused by the internal fault. 

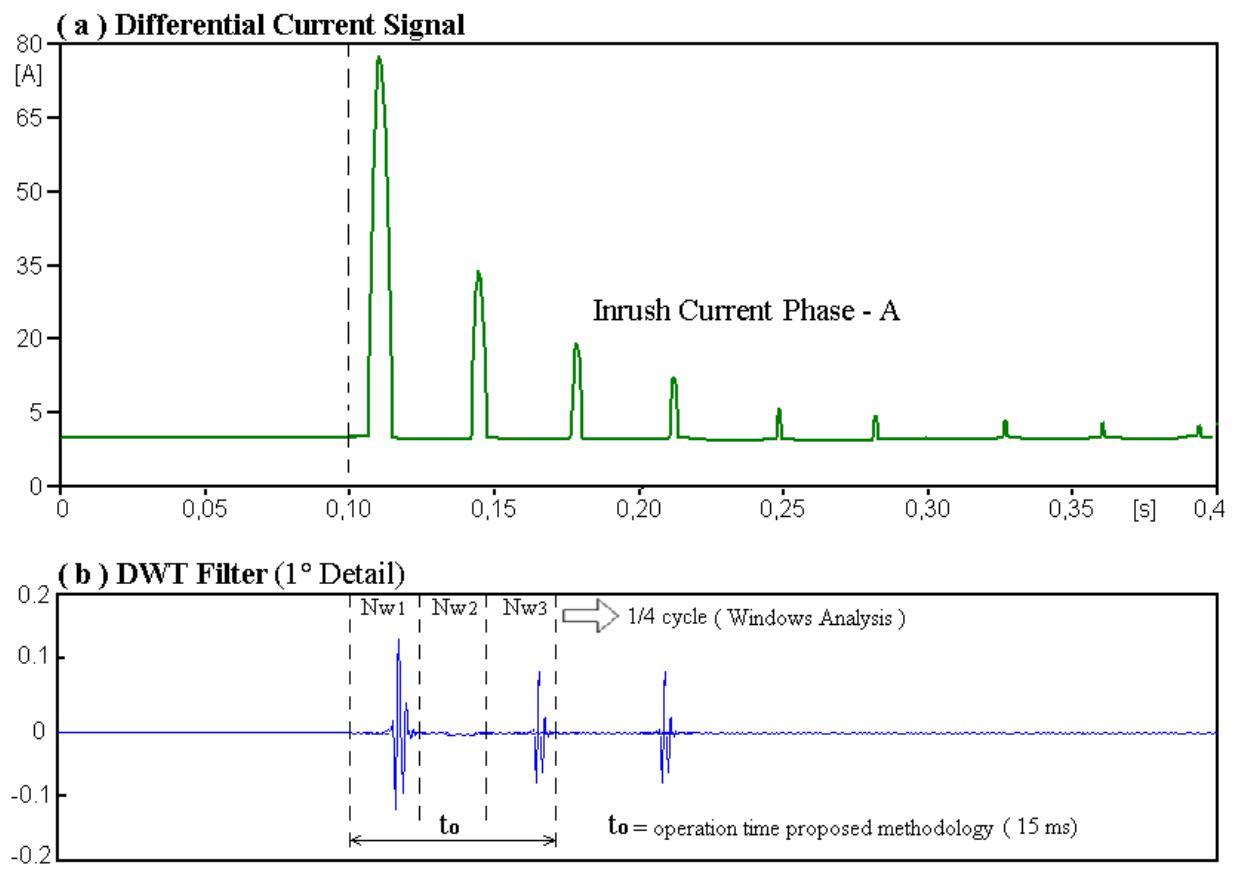

\section{(c) Signal Relay}

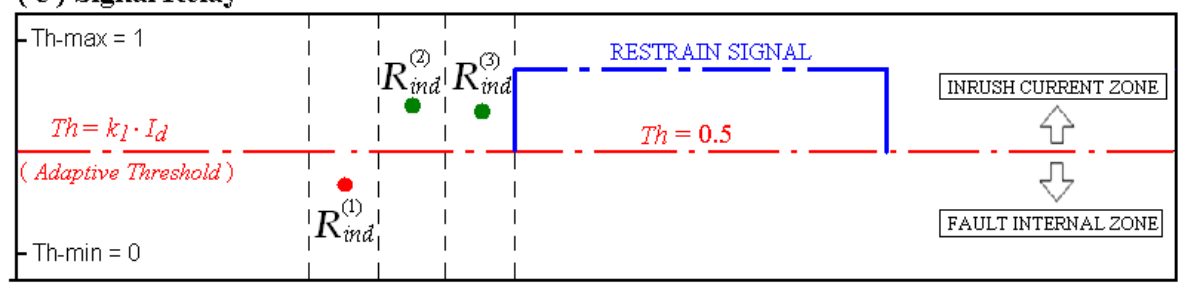

Fig. 10. Logical decision of the proposed algorithm to energization phenomenon.

\subsection{Obtained results}

The magnitude and shape of inrush current changes depending on several factors such as energization instant, core remnant flux, saturation of CTs and non-linearities of transformer core. However, in this work only the switching instant was evaluated. 12 energization cases were simulated for each switching angle and evaluated with the following mother wavelet: Daubechies (Db), Harr (Hr), Symlet (Sy), Coiflet (Coif) and Morlet (Mo).

Table 2 shows the proposed algorithm performance in correct operation number (OC[\%]) for transformer energization. In test development, the Daubechies mother wavelets presented the best performance for all switching angles with $97.11 \%$ correct diagnosis. The Harr mother wavelet type appeared as the least efficient with $18.75 \%$ of correct diagnosis. Furthermore, at $90^{\circ}$ switching angle presented the worse energization condition because it was the least correctly identified $(56.66 \%)$. However, others switches angles tested did presented a significant effect on the inrush current identification. 


\begin{tabular}{|c|c|c|c|c|c|c|}
\hline \multirow{2}{*}{$\begin{array}{c}\text { Switch } \\
\text { Angle }\end{array}$} & \multicolumn{5}{|c|}{ Mother Wavelet Type } & \multirow{2}{*}{ OC $[\%]$} \\
\hline & $\mathrm{Db}$ & $\mathrm{Hr}$ & Sy & Coif & Mo & \\
\hline $0^{\circ}$ & 12 & 7 & 12 & 12 & 12 & 91.66 \\
\hline $30^{\circ}$ & 12 & 2 & 12 & 12 & 12 & 83.33 \\
\hline $60^{\circ}$ & 12 & 0 & 10 & 12 & 10 & 73.33 \\
\hline $90^{\circ}$ & 11 & 0 & 9 & 8 & 6 & 56.66 \\
\hline $\mathrm{OC}[\%]$ & 97.11 & 18.75 & 89.58 & 91.66 & 85.41 & \\
\hline
\end{tabular}

Table 2. Performance of the proposed algorithm in percentage of correct operation (OC) [\%] to different switching instants.

Table 3 summarizes the methodology efficiency in percentage of correct operation of the proposed algorithm for different internal faults types and different fault resistances (RF). The performance was evaluated considering a constant load of 10 MVA on the end of the transmission line. There was an important drop in accuracy of the protection algorithm to internal fault cases in faults type A-B and A-B-C. However, the discrimination of faults type A-G (phase-ground) and A-B-G showed little sensitivity to $R_{\mathrm{f}}$ variation.

It was noted that the mother wavelet Daubechies showed an excellent performance and high efficiency in discrimination of simulated disturbances. This is because the decomposition solutions using Daubechies wavelet function are orthogonal and no marginal overlaps will happen during the signal reconstruction. The mother wavelet Symlet and Coiflet presented a satisfactory performance with a greater efficiency than the Morlet type. On the other hand, the wavelet Haar type did not achieved a good performance, presenting many inaccuracies in the discrimination of all simulated disturbances.

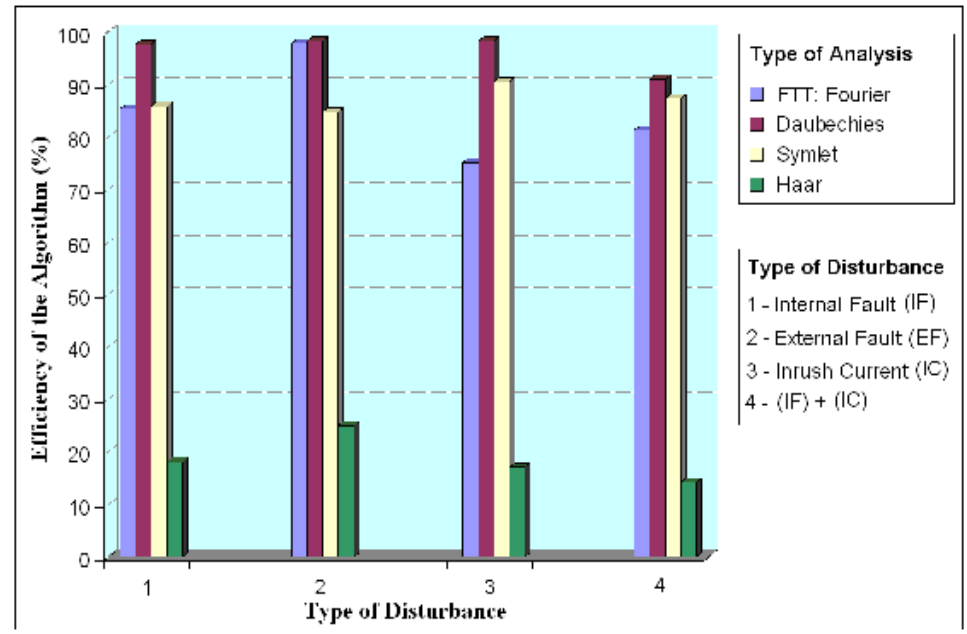

Fig. 11. Comparison between type wavelets functions and Fourier analysis (FTT).

To verify the wavelet function type effect on the proposed formulation, 3 wavelets function were compared with conventional protection methodology based in Fourier Analysis (FTT). The wavelet type used in the comparison study were: Daubechies, Haar and Symlet. The Fig. 11 shows the test results and the comparison between the proposed algorithm, a 
conventional percentage differential protection relay. It can be observed that the conventional technique based on FTT obtained a lower efficiency than the proposed algorithm.

\begin{tabular}{|c|c|c|c|c|c|}
\hline \multirow{2}{*}{$\begin{array}{l}\text { Mother } \\
\text { Wavelet }\end{array}$} & \multirow{2}{*}{$\mathbf{R}_{\mathrm{f}}[\Omega]$} & \multicolumn{4}{|c|}{ Internal Fault Type } \\
\hline & & A-G & A-B & A-B-G & A-B-C \\
\hline \multirow{4}{*}{$\mathrm{Db}$} & 0.01 & 100.0 & 100.0 & 100.0 & 100.0 \\
\hline & 10 & 100.0 & 100.0 & 100.0 & 100.0 \\
\hline & 50 & 99.22 & 98.28 & 100.0 & 100.0 \\
\hline & 100 & 98.90 & 97.66 & 98.44 & 100.0 \\
\hline \multirow{4}{*}{$\mathrm{Hr}$} & 0.01 & 82.36 & 81.65 & 83.15 & 84.15 \\
\hline & 10 & 76.32 & 76.54 & 75.18 & 75.36 \\
\hline & 50 & 72.65 & 71.54 & 73.21 & 73.26 \\
\hline & 100 & 70.18 & 69.32 & 70.15 & 70.15 \\
\hline \multirow{4}{*}{ Sy } & 0.01 & 99.38 & 100.0 & 100.0 & 100.0 \\
\hline & 10 & 98.75 & 98.75 & 99.68 & 100.0 \\
\hline & 50 & 97.81 & 97.65 & 98.75 & 98.75 \\
\hline & 100 & 97.18 & 97.03 & 98.12 & 95.75 \\
\hline \multirow{4}{*}{ Coif } & 0.01 & 100.0 & 100.0 & 100.0 & 100.0 \\
\hline & 10 & 99.38 & 98.75 & 100.0 & 97.34 \\
\hline & 50 & 98.75 & 91.25 & 97.50 & 92.81 \\
\hline & 100 & 97.65 & 87.66 & 96.87 & 88.28 \\
\hline \multirow{4}{*}{ Mo } & 0.01 & 97.21 & 96.54 & 94.65 & 94.36 \\
\hline & 10 & 96.24 & 95.64 & 95.63 & 94.62 \\
\hline & 50 & 95.12 & 96.35 & 94.32 & 93.12 \\
\hline & 100 & 90.15 & 84.71 & 85.63 & 89.34 \\
\hline
\end{tabular}

Table 3. Performance of the proposed algorithm to internal fault cases.

\section{Conclusions}

In this chapter a novel formulation for differential protection of three-phase transformers, based on the differential current transient analysis is proposed. The algorithms performance is evaluated using fault simulations in a typical electrical system under BPA's ATP/EMTP software. The algorithm considers the different magnitudes assumed by the DWT coefficients, induced by internal faults and inrush currents. The wavelet decomposition allows good time and frequency precision to characterize the transient events.

The proposed algorithm is comprehensible and feasible for implementation showing a correct operation with the adaptive threshold value. The obtained results through various simulated fault cases and non-fault disturbances showed that the proposed algorithm is robust and accurate.

Based on these tests and after critical evaluation of the proposed protection algorithm important conclusions could be observed:

- The use of Wavelet Transforms to analyze differential signals produced by transient phenomenon proved to be an effective and robust tool. 
- The variation of wavelets spectral energy coefficients proved to be an effective measure of discrimination.

- The proposed algorithm presents a perspective of practical application given the simplicity under which the methodology is based.

- The performance comparison made between the wavelet types: Daubechies (Db), Harr (Hr), Symlet (Sy), Coiflet (Coif) and Morlet (Mo), showed that the use of the Daubechies wavelet is the most appropriated.

- The comparative study with the conventional differential protection algorithm showed that the proposed formulation presents greater performance.

\section{References}

Abed, N. Y.; Mohammed, O. A. (2007). Modeling and Characterization of Transformer Internal Faults Using Finite Element and Discrete Wavelet Transforms. IEEE Transaction on Magnetics, Vol. 43, No. 4, (April 2007), pp. 1425-1428, ISSN 0018-9464.

Aggarwal, R.; Kim, C. H. (2000). Wavelet Transform in Power System: part 1 -general introduction to the wavelet transform. Power Engineering Journal, Vol. 14, No. 2, (April 2007), pp. 81-87.

Anderson, P. M. (1999). Power System Protection, Wiley-Interscience: IEEE Press with McGraw-Hill. ISBN 0-7803-3427-2, New Jersey, USA.

Bentley, P. M.; McDonnell, J. T. E. (1994). Wavelets Transform: an introduction. IEE Electronic \& Communication Engineering Journal, Vol. 6. No. 4 (August 1994), pp. 175186, ISSN 0954-0695.

Bonneville Power Administration. (2002). Alternative Transient Programs: ATP/EMTP. Retrieved from: http://www.emtp.rog/.

Daubechies, I. (1990). The Wavelet Transform, Time-Frequency Localization and Signal Analysis. IEEE Transactions on Information Theory, Vol. 36, No. 5 (September 1990), pp. 961-1005, ISSN 0018-9448.

Eissa, M. M. (2005). A Novel Digital Directional Transformer Protection Technique Based on Wavelet Packet. IEEE Transactions on Power Delivery, Vol. 20, No. 3, (July 2005), pp. 1830-1836, ISSN 0885-8977.

Faiz, J.; Lotfi-Fard, S. (2006). A Novel Wavelet-Based Algorithm for Discrimination of Internal Faults From Magnetizing Inrush Currents in Power Transformer. IEEE Transactions on Power Delivery, Vol. 21, No. 4, (October 2006), pp. 1989-1996, ISNN 0885-8977.

Harlow, J. H. (2007). Electric Power Transformer Engineering (2nd Edition), CRC Press Taylor \& Francis Group. ISBN 10-0-8493-9186-5, Boca Raton, USA.

Haykin, S.; Veen, B. V. (2001). Signals and Systems (2nd Edition), Jhon Wiley \& Sons Inc. ISBN 8-57-307741-7, Porto Alegre, Brazil.

Horowitz, S. H.; Phadke, A. G. (2008). Power System Relaying (3nd Edition), Ed. Research Studies Press Ltd. ISBN 978-0-470-05712-4, Baldock, England.

Mallat, S. (1999). A Wavelet Tour of Signal Processing (2nd Edition), Academic Press. ISBN 012-466606-X, California, USA.

Mao, P. L. ; Aggarwal, R. K. (2000). A Wavelets Transform Based Decision Making Logic Method for Discrimination Between Internal Faults and Inrush Currents in Power Transformer. International Journal of Electrical Power and Energy Systems, Vol. 22. No. 6 (August 2000), pp. 389-395, ISSN 0142-0615. 
Megahed, A. I.; Ramadan, A.; ElMahdy, W. (2008). Power Transformer Differential Relay Using Wavelet Transform Energies, Proceedings in the Power and Energy Society General Meeting IEEE, pp. 1-6, ISBN 978-1-4244-1905-0, Pittsburgh, USA, July 20-24, 2008.

Morate, M. G.; Nicoletti, D. W. (1999). A Wavelet-Based Differential Transformer Protection. IEEE Transactions on Power Delivery, Vol. 14, No. 4, (November 1999), pp. 1351-1358, ISSN 0885-8977.

Ngaopitakkul, A.; Kunakorn, A. (2006). Internal Faults Classification in Transformer Windings Using Combination of Discrete Wavelet Transform and BackPropagation Neural Networks. International Journal of Control, Automation, and Systems, Vol. 4. No. 3, (June 2006), pp. 365-371, ISSN 1598-6446.

Phadke, A. G. ; Thorp, J. S. (2009). Computer Relaying For Power System (2nd Edition), Ed. Research Studies Press Ltd. ISBN 978-0-470-05713-1, Baldock, England.

Polikar, R. (1999). The Story of Wavelets, Physics and Modern Topics in Mechanical and Electrical Engineering, World Scientific and Eng. Society Press, pp. 192-197, USA, Retrieved from: http://www.public.iastate.edu/ rpolikar.

Rioul, O.; Vetterli, M. (1991). Wavelets and Signal Processing. IEEE Signal Processing Magazine, Vol. 8. No. 4 (October 1994), pp. 14-38, ISSN 1053-5888.

Robertson, D. C. ; Camps, O. I. ; Mayer, J. S. ; Gish, W. B. (1996). Wavelets and Electromagnetic Power System Transient. IEEE Transactions on Power Delivery, Vol. 11, No. 2, (April 1996), pp. 1050-1058, ISSN 0885-8977.

Saleh, S. A.; Rahman, M. A. (2005). Modeling and Protection of Three-Phase Power Transformer Using Wavelet Packet Transform. IEEE Transactions on Power Delivery, Vol. 20, No. 2 (April 2005), pp. 1273-1282, ISSN 0885-8977.

Sarkar, T. K.; Su, C. (1998). A Tutorial on Wavelets from an Electrical Engineering Perspective, Part 2: The Continuous Case. IEEE Antennas and Propagation Magazine, Vol. 40, No. 6 (December 1998), pp. 36-49, ISSN 1045-9243.

Simpson, D. M. (1993). An Introduction to the Discrete Orthogonal Wavelet Transform. Revista Brasiliera de Engenharia, Vol. 9, No. 1, (July 1993), pp. 57-81.

The Mathworks Inc. (2010). Mathworks Matlab. Retrieved from: http://www.mathworks.com/

Thomas, W.; Ozgönenel, O. (2007). Diagnostic of transformer internal faults through ANN based on radial basis functions and dynamical principal component analysis. IET Generation,Transmission \& Distribution, pp. 1-11.

Vetterli, M.; Herley, C. (1992). Wavelets and Filter Banks: Theory and Design. IEEE Transactions on Signal Processing, Vol. 40, No. 9, (September 1992), pp. 2207-1992, ISSN 1053-587X.

Wang, H.; Butler, K. L. (2001). Finite Element Analysis of Internal Winding Faults in Distribution Transformer. IEEE Transaction on Power Delivery, Vol. 16, No. 3 (July 2001), pp. 422-428, ISSN 0885-8977.

Wiszniewski, A.; Kasztenny, B. (1995). A Multi-Criteria Differential Transformer Relay Based on Fuzzy Logic. IEEE Transaction on Power Delivery, Vol. 10, No. 4 (October 1995), pp. 1786-1792, ISSN 0885-8977.

Zaman, M. A.; Hoque, M. A.; Rahman, M. A. (1996). On-line Implementation of the Artificial Neural Network Based Protection for Power Transformer, Proceedings of NECEC, pp. 5-11, NL, Canada, May 17-22, 1996. 


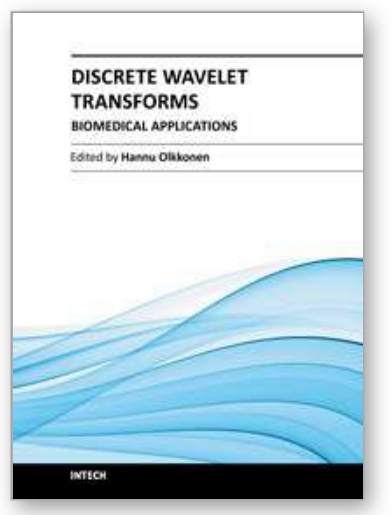

\author{
Discrete Wavelet Transforms - Biomedical Applications \\ Edited by Prof. Hannu Olkkonen
}

ISBN 978-953-307-654-6

Hard cover, 366 pages

Publisher InTech

Published online 12, September, 2011

Published in print edition September, 2011

The discrete wavelet transform (DWT) algorithms have a firm position in processing of signals in several areas of research and industry. As DWT provides both octave-scale frequency and spatial timing of the analyzed signal, it is constantly used to solve and treat more and more advanced problems. The present book: Discrete Wavelet Transforms - Biomedical Applications reviews the recent progress in discrete wavelet transform algorithms and applications. The book reviews the recent progress in DWT algorithms for biomedical applications. The book covers a wide range of architectures (e.g. lifting, shift invariance, multi-scale analysis) for constructing DWTs. The book chapters are organized into four major parts. Part I describes the progress in implementations of the DWT algorithms in biomedical signal analysis. Applications include compression and filtering of biomedical signals, DWT based selection of salient EEG frequency band, shift invariant DWTs for multiscale analysis and DWT assisted heart sound analysis. Part II addresses speech analysis, modeling and understanding of speech and speaker recognition. Part III focuses biosensor applications such as calibration of enzymatic sensors, multiscale analysis of wireless capsule endoscopy recordings, DWT assisted electronic nose analysis and optical fibre sensor analyses. Finally, Part IV describes DWT algorithms for tools in identification and diagnostics: identification based on hand geometry, identification of species groupings, object detection and tracking, DWT signatures and diagnostics for assessment of ICU agitation-sedation controllers and DWT based diagnostics of power transformers. The chapters of the present book consist of both tutorial and highly advanced material. Therefore, the book is intended to be a reference text for graduate students and researchers to obtain state-of-the-art knowledge on specific applications.

\title{
How to reference
}

In order to correctly reference this scholarly work, feel free to copy and paste the following:

Mario Orlando Oliveira and Arturo Suman Bretas (2011). Application of Discrete Wavelet Transform for Differential Protection of Power Transformers, Discrete Wavelet Transforms - Biomedical Applications, Prof. Hannu Olkkonen (Ed.), ISBN: 978-953-307-654-6, InTech, Available from:

http://www.intechopen.com/books/discrete-wavelet-transforms-biomedical-applications/application-of-discretewavelet-transform-for-differential-protection-of-power-transformers

\section{INTECH}

open science | open minds

\author{
InTech Europe \\ University Campus STeP Ri
}

\section{InTech China}

Unit 405, Office Block, Hotel Equatorial Shanghai 
Slavka Krautzeka 83/A

51000 Rijeka, Croatia

Phone: +385 (51) 770447

Fax: +385 (51) 686166

www.intechopen.com
No.65, Yan An Road (West), Shanghai, 200040, China 中国上海市延安西路65号上海国际贵都大饭店办公楼 405 单元 Phone: $+86-21-62489820$

Fax: $+86-21-62489821$ 
(C) 2011 The Author(s). Licensee IntechOpen. This chapter is distributed under the terms of the Creative Commons Attribution-NonCommercialShareAlike-3.0 License, which permits use, distribution and reproduction for non-commercial purposes, provided the original is properly cited and derivative works building on this content are distributed under the same license. 